\title{
El sentido en Deleuze a partir de la fenomenología
}

JUAN IGNACIO CháVEZ

Pontificia Universidad Católica del Perú

Resumen: El siguiente trabajo busca reflexionar sobre la génesis de la noción deleuziana de sentido. Se comenzará por demostrar que el proyecto de Deleuze, como el de Husserl, busca revertir el platonismo a partir del concepto de inmanencia. En segundo lugar, se hará una crítica de la noción de noema tal como está expuesta en Ideas I, con el fin de elucidar los rasgos que Deleuze pretende reformular: el buen sentido y el sentido común. Por último, se construirá sobre la crítica previa una descripción del sentido como acontecimiento.

Palabras clave: sentido, Deleuze, Husserl, noema, acontecimiento

Abstract: This paper intends to reflect on the genesis of the deleuzian notion of sense. I will start by demonstrating that Deleuze's project, as well as Husserl's, tries to revert Platonism by means of the concept of immanence. Secondly, I will criticise the concept of noema as exposed in Ideas I, in order to elucidate the features that Deleuze seeks to reformulate: good sense and common sense. Finally, based on the previous critique, I will carry out a description of sense as event.

Keywords: sense, Deleuze, Husserl, noema, event 


\section{§ 1. Introducción}

Las dificultades con las que parte una investigación sobre el sentido en ambos autores en cuestión son varias. La más inmediata consiste en que Husserl, por un lado, tiene una obra vertiginosamente cambiante, tanto en un sentido terminológico como en términos estrictamente filosóficos (aunque más adelante argumentaré que son lo mismo), sin mencionar la cantidad de manuscritos de investigación póstumos que son editados y traducidos hasta el día de hoy. Deleuze, por su parte, es un autor cuya intuición principal, aquella del pensamiento como rizoma, como recorrido descentrado e impredecible, dificulta cualquier comparación conceptual con autores que no tienen tal finura de matices.

Es por eso que me he propuesto centrar la investigación en Ideas relativas a una fenomenología pura y filosofía fenomenológica (1913), obra en la que Husserl descubre el terreno trascendental y con él la noción de noema o sentido, y Lógica del sentido (1969), primer intento de Deleuze por abordar temáticamente el sentido, si bien no de manera sistemática, sí por medio de múltiples series de pensamiento que logran dibujar un prisma suficientemente detallado.

El trabajo tiene el espíritu de exponer de manera introductoria la filosofía de Deleuze, a partir de una confrontación con la noción de sentido en Husserl. En otras palabras, es una reflexión crítica que ayuda no tanto a profundizar sobre alguno de los autores, sino a tratar de comprender la lógica de las reformulaciones que los unen. Eso nos permitirá no solo avizorar las nuevas posibilidades que las bases fenomenológicas permiten, sino también reflexionar sobre qué es lo que hace de la fenomenología un método sujeto a reformulaciones tan fundamentales que, sin embargo, no traicionan su impronta primordial.

\section{§ 2. El proyecto de Deleuze a partir de la fenomenología}

Según Leonard Lawlor, hay tres puntos en común entre Deleuze y la fenomenología: a) la reversión del platonismo; b) la inmanencia como trascendental 
pero no trascendente; c) la relación fundante es paradójica'. Antes de ver en qué sentido Deleuze busca hacer estas tres tareas, y con ello confirmar la comparación, sería bueno comenzar por explicar y discutir en qué sentido puede decirse que Husserl busca lograr dichos objetivos con la fenomenología. En primer lugar, con respecto de la inversión del platonismo, dice Lawnor que ello se debe a que Husserl plantea una epojé que reduce (reduces) el mundo trascendente a fenómeno ${ }^{2}$. Si bien es cier to que la fenomenología abandona una tarea metafísica icómo se da eso propiamente? A mi parecer, tan solo en este primer punto existe el riesgo de pasar por alto el carácter metodológico de la epojé del mundo de la vida y de la reducción fenomenológico-trascendental. Buena parte de los problemas entre Husserl y sus contemporáneos se debió a la interpretación de lo que podríamos llamar una exacerbación del terreno de la inmanencia ${ }^{3}$, es decir, el olvido de que, si bien es cierto que el fenomenólogo tiene frente a sí sentidos intencionalmente constituidos, y por lo tanto, inmanentes, la inspección fenomenológica no se agota en una dilucidación del cómo del aparecer del mundo, sino en la ulterior trascendencia de ese mundo real, es decir, siempre con miras a volver a la tesis de realidad. Más bien, podría decirse que cuando Husserl habla de esencias morfológicas, o de esencias exactas-que deben distinguirse del sentido-, está teniendo un gesto platónico, no en cuanto postulación de existencia de entidades reales ajenas al mundo, pero sí en cuanto postulando posibilidades reales en un sentido amplio, no reales en cuanto naturales, pero sí reales en cuanto determinan nuestra experiencia ${ }^{4}$.

El segundo punto, el de la reducción a una inmanencia trascendental, es quizá la contracara de la reversión platónica, y trata básicamente de la reducción del mundo a la inmanencia que lleva a cabo Husserl. Sobre esto habría que acotar, antes de hablar de Deleuze, que lo que Husserl está haciendo cuando

I Cf. Lawlor, Leonard, "Phenomenology, metaphysics, and chaos: of the fragility of the event in Deleuze”, en: Cambridge Companion to Deleuze, New York: Cambridge University Press, 2012, p. 103.

2 Cf. ibid.

3 Buena parte del problema se debió al uso por parte de Husserl del término "idealismo trascendental", el cual hacía que sus contemporáneos confundieran su filosofía con la kantiana o, incluso en algunos casos, con la berkeleyana.

4 Cf. Husserl, Edmund, Ideas relativas a una fenomenología pura y una filosofía fenomenológica, traducción de Antonio Zirión, México D.F.: Fondo de Cultura Económica, 20I3, §3. En adelante Ideas I. 
descubre el terreno puro de la consciencia intencional no es reducir el mundo trascendente a una inmanencia, sino descubrir que al sujeto le es inmanente el modo en que lo trascendente se le presenta. Es cierto que lo psicológico, o lo noético en cuanto ingrediente es también inmanente, pero lo mismo valdría para el estatuto moderno de lo psicológico, por lo que eso no es una novedad. La importancia del descubrimiento de la inmanencia de Husserl radica en la identificación de un terreno irreal que, aunque no es productivo ${ }^{5}$, es objetivo y necesario para que el ser humano comprenda su experiencia. Este terreno objetivo, a saber, el noema, es comparable solo con el objeto intencional previo a la simplificación epistemológica que el empirismo llevó a cabo en la modernidad, es decir, aquel que Descartes heredó de la escolástica. Es un terreno intermedio entre el sujeto y el mundo real.

El tercer punto, aquel que dice que la relación fundante en la fenomenología es paradójica, se refiere a que el suelo de la experiencia (lo fundante) permanece en la experiencia (se asemeja a lo fundado), y sin embargo se distingue de ella. El significado del adjetivo "paradójico" que describe a esta fundamentación no debe ser tomado por "circular" o "contradictorio", de hecho, el adjetivo resulta un halago por parte de Deleuze, pues este modo de fundamentación es quizá el elemento más importante que él recoge de Husserl, y buena parte de por qué puede decirse que su pensamiento parte de la fenomenología. Una comparación con la fundamentación metafísica puede ayudarnos a rodear esta idea: si pensamos, por ejemplo, en el modo en que un primer motor causa el mundo, o en que una sustancia pensante fundamenta el conocimiento de una sustancia extensa (a partir de Dios, en el caso de Descartes), podemos ver que en ambos casos existe un hiato entre lo fundante y lo fundado que imposibilita la inteligibilidad de la relación. Ese fue, quizá, el problema que enfrentaron los modernos y lo que para los antiguos pasó desapercibido. El caso de Husserl es distinto: para él, lo trascendente, el mundo real que experimentamos es una constitución en la experiencia, no por una sustancia pensante que se distingue del mundo, como en Descartes, sino por una relación intencional, es decir, por una estructura constituyente que se halla en el terreno de la experiencia misma. $Y$ es justamente por este sentido 
paradójico que, en términos de Deleuze, sí podría decirse sin problemas que la fenomenología comporta una crítica al platonismo.

Deleuze también hace una crítica al platonismo, pero no únicamente en el sentido en que uno se imaginaría. En primer lugar, habría que decir sobre esto que para Deleuze, la metafísica no se reduce a la metafísica platónica, o a la metafísica de la representación, lo cual sí hace, por ejemplo, Heidegger ${ }^{6}$, quien sienta su filosofía en una recuperación de principios pre-metafísicos de los filósofos presocráticos frente a la comprensión del ser como ente en Platón. Es por esto que, una vez que Deleuze retoma la impronta nietzschena formulada en El nacimiento de la tragedia de la "inversión del platonismo", lo hace a partir de la construcción de otra "imagen del pensamiento", en buena cuenta, de otra metafísica. Si bien es cierto que busca destituir la noción de "esencia trascendente", para Deleuze el platonismo no consiste únicamente en la distinción entre realidad esencial y apariencia, sino también, como desarrolla Platón en el décimo libro de La República, en la distinción entre apariencia y simulacro, es decir, entre lo que depende ontológicamente de la idea, y lo que logra escapar de su determinación y permanecer en total caos, respectivamente. Es así que la filosofía de Deleuze tiene una lucha por un doble frente: por una lado critica a la metafísica representacional, por otro critica el caos, lo cual muchas veces es pasado por alto ante una lectura desatenta de su obra.

En cuanto al modo en que lleva esto a cabo, habría que comenzar por decir que para criticar al platonismo, Deleuze "elabora una metafísica naturalista e inmanente nutrida por una ontología de lo virtual"7. En ella no hay nada trascendente, sino que todo lo que existe es parte de un mismo campo, tal como Spinoza entiende la realidad, un plano virtual en el que, sin embargo, todo se diferencia de todo. En otras palabras, dentro de un plano de inmanencia en donde todo es posible de la misma manera, se hace una diferencia entre el plano mismo en cuanto fundamento (virtualidad o posibilidad) y lo actualmente construido, pues de lo contrario no existiría más que un instante presente.

6 Cf. De Beistegui, Miguel, "The Deleuzian reversal of platonism”, en: Cambridge Companion to Deleuze.

7 Barroso, Moisés, Inmanencia, virtualidad y devenir en Gilles Deleuze, tesis doctoral, San Cristobal: Universidad de la Laguna, p. 39. 
Las dificultades de Deleuze -y sin dudas tiene más dificultades que Husserl en este aspecto- parten de su intento de demostrar que hay diferencia en la mismidad una vez que ha absolutizado la inmanencia ${ }^{8}$. La trascendencia viene a ser, más bien, todo lo absolutamente otro, lo que se resiste a ser construido por nosotros.

Finalmente, y tomando en cuenta esto último, la fundamentación inmanente en Deleuze es paradójica del mismo modo en el que Husserl pretende fundar la experiencia a partir del plano de la experiencia. Para Deleuze, el sentido (lo fundante) no existe fuera de la expresión, y sin embargo tampoco es un reflejo de ella. En otras palabras, pertenece al mismo campo, pero es totalmente novedoso. Pero si sabemos que Deleuze también maneja una filosofía paradójica, ¿en qué puede consistir esta crítica?

Lo que Deleuze intentará demostrar es que el carácter paradójico de la fundamentación del sentido en Husserl es solo aparente, y que si bien la fenomenología no es metafísica, sí tiene cosas en común con ella. En Lógica del sentido nos dice que la fenomenología entiende la inmanencia a partir de una "relación dativa" a un sujeto o una consciencia que constituye lo dado. $Y$ como habíamos dicho, el sentido, para ser propiamente inmanente, no debe ser reflejo de algo más, ni dejarse determinar por alguna instancia anterior y ajena a él ${ }^{9}$. Ante esto, lo que uno podría responder desde la fenomenología es lo siguiente: lo que logra Husserl con la reducción fenomenológica es justamente evitar que haya un sujeto sustancial que funde algo desde sí mismo, puesto que la estructura mínima a la que uno puede llegar es la conciencia intencional, es decir, una conciencia que no es nada si no es ya conciencia de algo. Pero aun así, podríamos seguir diciendo desde Deleuze que lo que logra el fenomenólogo cuando hace la epojé fenomenológico-trascendental no es acceder a una inmanencia paradójica, sino meramente retroceder desde el ámbito de la doxa, es decir, desde la actitud natural, al ámbito de la Urdoxa ${ }^{10}$.

8 Cf. ibid., pp. 40-4I.

9 Cf. Lawlor, Leonard, op. cit., p. 104.

10 Llamada también "certeza fundamental" por Dermot Moran (cf. Moran, Dermot, Husserl's dictionary, London/New York: Continuum, 2012, p. 86). 
Conviene explicar bien este último concepto: como sabemos, todas las vivencias intencionales son susceptibles de adecuación entre significación e intuición (síntesis de identificación), y por tanto de tener una historia de asentimientos subjetivos en el plano noemático. Es así que para cada uno de los objetos intencionados, conjuntamente con sus modos de creencia, siempre cuenta el cogito con una "creencia acerca de su modo originario de darse" ", una Urdoxa. De Nigris lo ejemplifica de este modo: “(...) a Don Quijote me lo puedo presentar como el amigo de Sancho o como el contrincante del Bachiller Sansón Carrasco, pero su modo de darse originario, que funda $a$ priori todos sus sentidos y cualidades aprehensivas posibles, es imaginativo. Si me dicen: "he visto a Don Quijote paseando por la calle", mi acto pondrá tal sentido con una doxa de duda, porque el estado de cosas (Sachverhalt) mencionado en ella se funda en la Urdoxa originaria que pone a Don Quijote como ser de ficción"'2.

Si entiendo correctamente la Urdoxa, ella es una creencia fundamental que está detrás del sentido, en cuanto lo funda. Ella tiene el papel de "la protoforma [Urform] 'no modalizada' de los modos de creencia. Por consiguiente, en el correlato, el carácter de ser pura y simplemente (el noemático ser 'cierto' o 'real') funciona como la protoforma de todas las modalidades de ser"'3. Siendo así, el sentido no es una fundamentación libre de su propio terreno, sino que resulta dependiente de una instancia fundante. El asunto, para Deleuze, se reduce a una cuestión de génesis del sentido. ¿Qué tan legítima es una génesis en la que el sentido es segundo con respecto de algo más? Si algún desafío tiene la inmanencia, es la génesis del sentido sin una remisión a un sujeto o a un dativo que funde retroactivamente.

\section{§ 3. La constitución de sentido}

Para Deleuze, el sentido es un "acontecimiento", y un acontecimiento está siempre en el lenguaje. Es por eso que el sentido debe siempre generar el

II De Nigris, F., "Intencionalidad, pasividad y autoconciencia en la fenomenología de Husserl” en: Ideas y Valores, No 64 (20I5), p. 226.

12 Ibid.

I3 Husserl, Edmund, op. cit., p. 335. 
elemento primario del discurso: la proposición ${ }^{14}$. Ella tiene tres funciones distintas: la denotación, la manifestación y la significación, o dicho de otro modo, el sentido debe generar: i) el estado de cosas denotado por la proposición (denotación), ii) los estados del sujeto manifestados por la proposición (manifestación), iii) los conceptos significados y las clases de proposición (significación).

La denotación viene a ser la utilización de palabras para la selección de imágenes particulares en relación de representación con el estado de cosas exterior (datum). Es una indicación que dice "es un esto", "no es un esto" y que está sujeta a veracidad o falsedad. La manifestación, por su parte, es la relación entre la proposición y el sujeto que la expresa, es decir, los deseos y creencias que la fundan. Tal como hubiese dicho Hume, Deleuze afirma que los deseos humanos son "inferencias causales", inferencias que desde ya nos mueven a alcanzar la imagen que tenemos en mente (en un sentido amplio) y volverla existente en el estado de cosas. Si bien desde un punto de vista prima la designación, es evidente que toda la libre asociación de palabras para representar el mundo como estado de cosas luce unilateral una vez que nos preguntamos por las causas de esa asociación. Una vez que pasamos del plano de la designación al de la manifestación, tal como se puede observar fácilmente en las Meditaciones Metafísicas de Descartes, "se produce un desplazamiento de valores lógicos representado por el Cógito [sic]: no ya lo verdadero y lo falso, sino la veracidad y el engaño"15. Lo importante está pues, en cómo el cogito funda la designación. En cuanto a la significación, es la "relación de la palabra con conceptos universales o generales y de las relaciones sintácticas con implicaciones de concepto" ${ }^{\prime 6}$. Su punto de vista es el de las conexiones de significados que están a la base de cualquier designación o manifestación. Si se demuestra algo del mundo, siempre hay conceptos que fungen de premisas y que terminan por hacer posible la conclusión. Esta vez hay un nuevo desplazamiento de los valores lógicos: ya no verdadero y falso, o veracidad y

14 Es pertinente hacer una aclaración: mientras que para Deleuze todo sentido es ya lenguaje, sabemos que para Husserl los noemas perceptivos son anteriores a los noemas de lenguaje, es decir, a los noemas predicativos. En este contexto, los gestos, por ejemplo, son solo expresiones de vivencias, no de predicaciones. El problema de si el lenguaje es co-originario con la percepción podría, sin embargo, tratarse de una ampliación del concepto de lenguaje en la tradición filosófica, y no realmente de una disputa conceptual entre Husserl y filósofos contemporáneos. Esta discusión, sin embargo, rebasa los límites de este trabajo.

I5 Deleuze, Gilles, Lógica del sentido, Buenos Aires: Paidos, 2005, p. I6. En adelante LS. 16 Ibid. 
engaño, sino condición de verdad y de falsedad, es decir, herramienta básica a partir de la cual se construyen los valores lógicos anteriores, como si antes de operar de cualquier modo con el lenguaje tuviese uno que sumergirse en un mar de significados conectados con los que cargar indefectiblemente. $\mathrm{Si}$ bien desde un punto de vista es la manifestación el centro fundante de toda significación ${ }^{17}$, desde otro punto de vista, es la significación la que funda toda interioridad del manifestante, o incluso se desarrolla por ella misma, independientemente del sujeto, en el plano de la lengua ${ }^{18}$.

Sin embargo, parece ser que la proposición no se puede apoyar completamente en la significación, o en sus condiciones mismas de verdad. Según Deleuze, cuando utilizamos la palabra "luego" en un silogismo y llegamos a parar en una conclusión, dejamos de lado las premisas y afirmamos la conclusión por sí misma, es decir, nos desentendemos de las premisas y las relaciones lógicas que la llevaron a ser aseverada en un primer lugar. E incluso si pudiésemos establecer la veracidad (en relación con el estado de cosas) de las premisas para que la conclusión se desprenda de ellas, habría que agregar una proposición adicional que vele por que el orden de implicación sea válida. Deleuze lo explica de este modo: “(...) incluso suponiendo que las premisas A y B sean verdaderas, de ellas no podemos concluir la proposición $Z$ en cuestión, no podemos desligarla de sus premisas y afirmarla por sí con independencia de las implicación, más que admitiendo que es, a su vez, verdadera si A y B son verdaderas: lo que constituye una proposición C que continúa dentro del orden de la implicación, que no alcanza a salir de él, ya que remite a una proposición $D$, que dice que $Z$ es verdadera si $A, B$ y $C$ son verdaderas... hasta el infinito (...). En resumen: con una mano se desliga la conclusión de las premisas, pero a condición de que, con la otra mano, se añadan siempre otras premisas de las que la conclusión no puede desligarse"19.

17 El punto de vista de la manifestación puede bien estar emparentado en la concepción de lengua como habla. Sin desmerecer el hecho de que, efectivamente, no existe tal cosa como una lengua fuera del habla, al plantear la independencia de la función significativa de la proposición, Deleuze hace un esfuerzo por limitarse a mostrar una relación real de funcionamiento, más que una postulación de alguna sustancia separada.

I8 Este es un recurso explicativo de Deleuze. No quiere decir en ningún sentido que pueda existir lengua sin hablantes.

19 LS, p. 18. 
Una vez que nos damos cuenta de que, no solo la designación y la manifestación dependen de la significación, sino que la función de la significación también plantea problemas de fundamentación, sabemos que estamos en lo que Deleuze llama el "círculo de la proposición"20. Para "hender el círculo" es necesario disponer de un "elemento propio distinto de la forma de lo condicionado, tener algo incondicionado capaz de asegurar una génesis real de la designación y de las otras dimensiones de la proposición”21.

¿Pero hay algo aparte de estas tres funciones de la proposición mencionadas? La respuesta de Deleuze es que más allá de lo designado, manifestado y significado en la proposición, hay tal cosa como lo expresado: el sentido, la cuarta dimensión de la proposición. En Lógica del sentido incursiona en este término a partir de un estudio de la ontología estoica. Los estoicos comprendieron la expresión como el Aliquid, el acontecimiento incorporal que insiste ${ }^{22}$ en la superficie de las cosas. Este Aliquid es irreductible a la proposición y sus términos, a las creencias personales y a los conceptos generales, pues tal como decían los estoicos, este no es "palabra, ni cuerpo, ni representación sensible, ni representación racional" 23 . Es así que, si bien el sentido produce los modos de la proposición, debe ser "neutral" con respecto de ellos. Deleuze encuentra estas características en el noema husserliano. Husserl distingue el noema perceptivo del objeto físico, la vivencia psicológica, las representaciones mentales y los conceptos lógicos, lo presenta como un incorporal. Además, el noema tiene la característica de no existir fuera de la expresión que lo expresa, es decir, de ser irreal, o en término deleuzianos, un extra-ser. Es así que termina por preguntarse: "iserá la fenomenología esa ciencia rigurosa de los efectos de superficie?" 24

Para responderse esta pregunta Deleuze investiga el texto en el que Husserl descubre el terreno del noema: Ideas relativas a una fenomenología pura y una filosofía fenomenológica. En vista de loya dicho, el noema, al producir los modos

20 Ibid.

21 Ibid., p. 19.

22 Deleuze utiliza indistintamente las palabras "insiste" $y$ "subsiste", pero dado que su punto es justamente que los incorporales son un extra-ser, y por tanto no sustanciales, creo que el segundo término puede llevar a confusiones. 
de la proposición y aun así no asemejarse a ninguno de ellos, parece cumplir con lo que Deleuze llamaría la propia paradoja de una génesis del sentido. Lo que Deleuze intentará demostrar en los primeros capítulos de Lógica del sentido es que esto se da solo en apariencia.

La primera crítica que Deleuze tiene sobre el noema se dirige específicamente a lo que Husserl denomina "núcleo". Para esto conviene revisar la distinción interior al noema entre los caracteres noemáticos y el núcleo que hace Husserl en Ideas I. Los caracteres noemáticos no son otra cosa que "carácter de 'posición', caráter ‘tético' en el sentido más amplio”25, es decir, los caracteres de creencia subjetivos que se presentan como correlatos de los modos de vivencia en el polo noético. Es lo que en Investigaciones Lógicas se llama 'cualidad'26. El núcleo, en cambio, es el contenido del noema, aquello que apunta o se dirige hacia el objeto trascendente. Así como la morphé intencional es lo estrictamente noético, puede decirse que el núcleo, o la "materia", como se le denomina en Investigaciones Lógicas, es lo estrictamente noemático. Este núcleo contiene, por un lado, propiedades del objeto cuya descripción define el modo en que se da cada vez, y por otro, el objeto mismo en cuanto portador de esas propiedades. Ese portador es el punto central del núcleo del noema, aquello de lo que se predica: el objeto o la X. Él permanece invariable a pesar de que en una experiencia particular pueda haber muchos predicados cambiantes que describir, es decir, el objeto es idéntico, pero el núcleo del noema es mudable. Es por esto, dirá Husserl, que dos diversos conceptos de objeto pueden ser distinguidos: el objeto como punto (soporte) central (la X) y el objeto en el cómo de sus determinaciones (el núcleo entero del noema). El sentido es justamente el objeto en esta segunda acepción que incluye las propiedades cambiantes. Cuando se tiene más de un sentido en enlace concordante, todos esos sentidos se refieren al mismo objeto y se sintetizan.

Ahora bien, la primera crítica al noema consiste en que Husserl entiende el núcleo "como predicado y no como verbo, es decir, como concepto y no como acontecimiento (por ello, según Huserl, la expresión produce una forma de lo conceptual, y el sentido es inseparable de un tipo de generalidad, aunque

25 Husserl, Edmund, op. cit., p. 396.

26 Cf. Husserl, Edmund, Investigaciones Lógicas, traducción de Morente y Gaos, Madrid: Alianza Editorial, 1982. 
esta generalidad no se confunda con la de la especie)"27. ¿Qué consecuencias tiene esto? En primer lugar, si nos movemos en el terreno de los conceptos, el núcleo pasa a ser lo que desde la perspectiva de Deleuze se entiende como significación, y su relación dadora de sentido opera de modo designativo con respecto de los objetos. Además, una vez que tenemos una serie de predicados, o un "contenido" de predicados, la operación natural es buscar la X del núcleo, es decir, el objeto trascendente que en este caso funge como principio de unificación de los predicados. Esa X, lejos de ser interior al sentido, dirá Deleuze, es el objeto kantiano, "donde $X$ significa 'cualquiera', en relación racional extrínseca de trascendencia con el sentido" 28 .

Vale la pena desarrollar el modo en el cual Deleuze, a partir de este primer argumento, plantea su crítica contra la filosofía trascendental en general. El error de Kant y Husserl, dice, es "concebir el trascendental a imagen y semejanza de lo que se pretende fundar" 29 . Kant no logra ver el espectro genético dentro de su fundamentación trascendental, por lo que la constitución de mundo es un directo condicionamiento de los fenómenos con respecto de las estructuras del sujeto. Husserl, por su parte, sí concibe una génesis del sentido, pero según Deleuze, a partir de un estrato "originario" perteneciente a la conciencia constituyente ${ }^{30}$. La justificación, en ambos casos, para la "originariedad" de la conciencia constituyente, radica en que, para que la filosofía trascendental funcione, se requiere una cláusula según la cual "las condiciones de los objetos reales del conocimiento deben ser las mismas que las condiciones del conocimiento"31. La forma objeto = xy la forma pura de la conciencia deben estar alineadas según una instancia anterior.

Si bien este modus operandi evita que los objetos tengan condiciones autónomas al sujeto, y que puedan concebirse potencialmente como esencias subsistentes en el sentido de la metafísica antigua, Deleuze cree que la fenomenología tiene algo en común con la metafísica, a saber, que ambas plantean una bifurcación

27 LS, p. 74.

28 Ibid.

29 Ibid., p. 80.

30 Si bien es cierto que la conciencia constituyente, según el aspecto genético de la fenomenología de Husserl, es producida históricamente, queda abierta la discusión de cómo justificar una teleología gnoseológica sin delimitar esa producción.

2231 lbid. 
radical del destino del pensamiento: o bien cae en "un fondo indiferenciado, sin-fondo, no-ser informe, abismo sin diferencias y sin propiedades; o bien [permanece] un Ser soberanamente individuado, una Forma fuertemente personalizada" ${ }^{2}$. La conciencia trascendental viene a ser, de este modo, el salvavidas que evita la irrupción de la singularidad, el hundimiento en el caos.

La segunda crítica al noema radica en que Husserl lo comprende explícitamente como una idea en sentido kantiano, es decir, comprende que toda síntesis de vivencias y, por lo tanto, de noemas, se construirá, por obra de corrección, a partir de una perfección pre-delineada. Si bien cualquier cambio de validez en la relación intencional es parte de la vida, o de la vida comunitaria (para extender la fenomenología en toda su extensión y no caer en la caricatura del fundacionalismo cartesiano), hay un principio de generalidad incondicionada que dice que "a todo objeto 'verdaderamente existente' corresponde la idea de una conciencia posible en la cual el objeto mismo es captable originariamente y además en forma perfectamente adecuada" ${ }^{33}$. Esta es la fundamentación gnoseológica que ya había mencionado más arriba: aquella de la Urdoxa, que Deleuze considerará promotora del "sentido común" y del "buen sentido" 34 .

Ahora bien, Deleuze entiende el sentido común y el buen sentido como dos aspectos de la doxa. El buen sentido es básicamente la dirección, sentido único, como cuando orientamos la flecha del tiempo, bien al futuro, bien al pasado, siempre respecto de un presente, es decir, "respecto de una fase determinada del tiempo escogida en el sistema individual considerado" ${ }^{35}$. Es condición para la previsión, pues sin una dirección determinada dentro de un sistema cualquiera, la gama de posibilidades se bifurca. Podría decirse también que es una dirección a partir de una determinación. Y, según Deleuze, su direccionalidad tiene consecuencias específicas:

"Los caracteres sistemáticos del buen sentido son pues: afirmación de una sola dirección; la determinación de esta dirección como yendo de

32 Ibid., p. 81.

33 Husserl, Edmund, Ideas I, p. 43I.

34 Es importante recalcar que, si bien la idea es adecuadamente captada, su contenido no. Es discutible, sin embargo, hasta qué punto eso podría deslindar la Urdoxa de lo que Deleuze llama "buen sentido".

35 LS, p. 59. 
lo más diferenciado a lo menos diferenciado, de lo singular a lo regular, de lo notable a lo ordinario; la orientación de la flecha del tiempo, del pasado al futuro, según esta determinación; el papel director del presente en esta orientación; la función de previsión que de este modo se hace posible; el tipo de distribución sedentaria en la que se reúnen prácticamente todos los caracteres precedentes" ${ }^{36}$.

Si es que el buen sentido resulta por ahora un concepto muy abstracto, definitivamente se aclara una vez que lo relacionamos con el sentido común. Este último, dice Deleuze, "subsume facultades diversas del alma u órganos diferenciados del cuerpo, y los remite a una unidad capaz de decir Yo", [y por otro lado] "subsume la diversidad dada y la remite a la unidad de una forma particular de objeto o de una forma individualizada de mundo" ${ }^{37}$. Es decir, es aquella facultad que me permite, por ejemplo, decir que lo que ahora toco, veo y pienso es este teclado de computadora, y al mismo tiempo que quien hace las labores de tocar, ver y pensar el teclado, soy yo. Podríamos decir que es un principio de unidad de las facultades y de los objetos que se relacionan con las facultades, o que es una facultad de mayor rango, en cuanto tiene la función de interrelacionar las facultades. Lo que es claro es que la lectura que Deleuze tiene sobre el sentido común es similar a la que tiene sobre el buen sentido, en cuanto a que ambos tienden a homogenizar, a remitir la diversidad a "la forma de lo Mismo"38.

La diferencia entre ambos radica en que mientras el sentido común identifica, reconoce, el buen sentido prevé, y no es muy difícil ver por qué son complementarios. Según Deleuze, el buen sentido solo puede asignar principio y fin, dirección, si se remite a una instancia capaz de ordenar la diversidad en la forma de un sujeto, de un objeto o un mundo. En otras palabras, sería imposible sustentar una teleología de la síntesis intersubjetiva del conocimiento si no hubiese, primero, un Yo de base. E inversamente, la subjetividad tendría un poder de organización vacío si no pudiese emprender un viaje de experiencia en alguna dirección determinada, determinando tal o cual diversidad ${ }^{39}$. Es así que, si nos remitimos concretamente a los términos técnicos de Ideas I,

36 Ibid., p. 60.

37 Ibid., p. 61.

38 Ibid.

2439 Cf. ibid. 
podemos ver que la Urdoxa es aquel sentido común, y la teleología es el buen sentido posibilitado por ella.

Habiendo explicado estos dos conceptos, es buen momento para intensificar nuestra paulatina aproximación a la noción de "sentido" en Deleuze. En Lógica del sentido lo presenta como aquello que no es buen sentido ni sentido común. En una palabra, como aquello paradójico:

"Lo propio del sentido es no tener dirección, no tener "buen sentido", sino siempre los dos a la vez, en un pasado-futuro infinitamente subdividido y estirado. El físico Boltzmann explicaba que la flecha del tiempo, yendo del pasado al futuro, solo valía en mundos o sistemas individuales, y respecto de un presente determinado en tales sistemas: "para el Universo entero las dos direcciones del tiempo son pues imposibles de distinguir, así como en el espacio no hay ni arriba ni abajo» (es decir, ni altura ni profundidad)" ${ }^{\prime 40}$.

Tal como da a entender esta analogía de corte físico, Deleuze comprende que el sentido no puede tener una dirección, ni un agente, pues tenerlos implicaría ya un punto de apoyo en el plano, un arbitrario poner el dedo sobre la línea infinita, en fin, una determinación sobre la cual no está dispuesto a negociar.

La tercera crítica hacia el noema tiene que ver con el modo en el cual Husserl asegura su cualidad de productivo y neutral, respectivamente. Habíamos dicho ya que, para Deleuze, el sentido produce los modos de la proposición, y aun así es neutral con respecto de ellos. Así explica esta neutralidad en Lógica del sentido:

"Respecto de los modos proposicionales en general, la neutralidad del sentido aparece desde varios puntos de vista. Desde el punto de vista de la cantidad, el sentido no es ni particular ni general, ni universal ni personal. Desde el punto de vista de la cualidad, es completamente independiente de la afirmación y de la negación. Desde el punto de vista de la modalidad, no es ni asertórico, ni apodíctico, ni siquiera interrogativo (modo de incertidumbre subjetiva o de posibilidad objetiva). Desde el punto de vista de la relación, no se confunde en la proposición que lo expresa ni con la designación, ni con la manifestación, 
ni con la significación. Finalmente, desde el punto de vista del tipo, no se confunde con ninguna de las intuiciones, de las «posiciones» de conciencia que pueden determinarse empíricamente gracias al juego de los caracteres proposicionales precedentes: intuiciones o posiciones de percepción, de imaginación, de memoria, de entendimiento, de voluntad empírica, etc" ${ }^{\prime \prime}$.

Para Husserl, "la conciencia en general, de cualquier índole y forma que sea, está cruzada por una radical distinción" ${ }^{2}$ : hay noesis que se ajustan a la jurisdicción de la razón, y noesis que no. Las primeras son las "propias" (conciencia posicional), las propiamente téticas, las que ponen algún tipo de posición sobre el objeto, como la creencia o la duda, y las segundas son las "impropias" (conciencia neutral), el "mero pensamiento" que no cabe reconocer como correcto o como incorrecto. La relación entre ambos tipos de actos consiste en que los propios se remite a un cogito real, mientras que el otro es solo la sombra de un acto, "el uno obra en realidad, el otro es el mero reflejo de un obrar"43. Si bien Husserl pide al lector no interpretar las expresiones figuradas de "sombra", "reflejo" o "imagen" como mera ilusión, queda clara la jerarquía entre ambos modos de la conciencia. Además, se encarga de dejar en claro que "la distinción entre posicionalidad y neutralidad no expresa (...) una mera particularidad referente a las posiciones de creencia [sino que] es de hecho una distinción universal de la conciencia" ${ }^{\prime 4}$.

La neutralidad del sentido, desde el punto de vista de la fenomenología de Husserl, no significa para Deleuze una neutralidad legítima. Lo que debiera ser característica del sentido por sí mismo: el ser neutral y productivo al mismo tiempo, son características respectivas de una disyunción artificial: la conciencia posicional (productiva) y la conciencia neutral.

4 Ibid., p. 77.

42 Husserl, Edmund, Ideas I, p. 354.

43 Ibid., p. 335.

$2644 \quad$ lbid., pp. 357-358. 


\section{§ 4. El acontecimiento}

El acontecimiento es singularidad, sentido. Para Deleuze, estas palabras se refieren a lo mismo y quizá solo iluminen aspectos distintos de aquello a lo que nos aproximamos. Husserl comprende el sentido como la capa expresiva (noemática) que acompaña siempre la tesis posicional o neutral que se experimenta en la expresión (noesis). Si bien hemos visto que esa capa expresiva se entiende comúnmente como significación, tal como él mismo acepta en §I24 de Ideas I, importa destacar otra parte del mismo parágrafo que Deleuze suscribe totalmente, a saber, que "la capa de la expresión -esto constituye su peculiaridad-no es productiva. $O$ si se quiere: su productividad, su rendimiento noemático, se agota en el expresar" 45 . Es de esta noción que Deleuze extrae dos características para su concepción de sentido: la esterilidad y la neutralidad. Por un lado, el sentido es estéril porque no produce, lo que explica que una capa expresiva no pueda tener una tesis distinta que aquella de la expresión. Por otro lado, como ya debe estar claro, es neutral, porque se distingue de lo psíquico, físico, conceptual, etc.

Sin embargo, mientras que para Husserl el sentido es una síntesis de predicados amparados por el sentido común y el buen sentido, para Deleuze el sentido es singularidad, es decir, pre-individual, impersonal, anónimo. No es más que un punto o un instante ${ }^{46}$. $Y$ del mismo modo en que un punto no tiene dimensión, y es a la vez todo y a la vez nada, la singularidad es lo que en la estructura del sentido deleuziana viene a llamarse el "elemento paradójico", lo que constituye el acontecimiento a través del cual están distribuidos todos los demás acontecimientos ${ }^{47}$. El elemento paradójico incluye tanto el sentido como el sinsentido, es como una pregunta que tiene demasiadas respuestas, pero ninguna correcta, ningún punto con alguna prerrogativa sobre los otros.

Como dije más arriba, la filosofía de Deleuze tiene una lucha por dos frentes: por un lado, con las esencias trascendentes (ulteriormente las ideas platónica), por otro, con el caos (ulteriormente los simulacros platónicos). Si nos volvemos a remitir a los estoicos, veremos que los incorporales son efectos de los

$45 \quad$ Ibid., p.384.

46 Cf. Lawlor, Leonard, op. cit., p. II0.

47 Cf. ibid. 
cuerpos. Son las mezclas de cuerpos las que causan todo lo que hay (o de lo contrario tendríamos un idealismo subjetivo), y los efectos incorporales, o en nuestros términos, los acontecimientos, son efectos de esas mezclas de cuerpos. Lo que en términos corporales es accidente de la sustancia, en términos incorporales es acontecimiento, efecto de superficie que no se toca ni con lo caótico de la mezcla de los cuerpos, ni se eleva hasta a nivel de idea platónica.

Nadie podría dudar, por ejemplo, de que hay un sustrato material para una herida. Pero lo que acontece con una herida no es, según Deleuze, un mero accidente, sino el insistir de la cicatriz, el referirse a un "ya no" de la totalidad de la piel como estaba antes, y a un "aún no" de la totalidad de la piel cuando la cicatriz se vaya. Lo material se convierte así en un acontecimiento ideal que se vuelve indiferente a los cuerpos ${ }^{48}$. Es así que los acontecimientos no son predicados, tal como entenderíamos el sentido a partir de Husserl, sino verbos. Verbos que nos enfrentan con sentidos perceptivos tales como el "verdear" de un árbol, más que el predicado "verde" relativo al árbol. Y es justamente el infinitivo del verbo lo que le da la potencia de dividirse infinitamente en cualquier tiempo. El verdear del árbol es la singularidad que experimentamos, pero que en cuanto acontece se eleva realmente a un nivel ideal de evento puro y neutral, tal como Husserl concebía el noema. Tan puro y neutral que, si bien el acontecimiento es siempre una novedad, al mismo tiempo coge un estatuto de "eterno", de sin origen y repetible para siempre, hacia ambos sentidos en el tiempo.

En Diferencia y repetición, Deleuze toma como ejemplo un extracto de En busca del tiempo perdido de Marcel Proust en que el protagonista tiene una reminiscencia (un recuerdo involuntario) del sabor de una magdalena en su pueblo natal, Combray. Si entendemos el presente como lo que puede ser, pudo ser, y podrá ser percibido, podemos diferenciar un presente pasado, aquel presente en que hubo alguna vez una magdalena siendo saboreada por él, $y$ un presente presente, aquel en que el autor evoca la magdalena y se la representa tal como podría ser saboreada en ese momento. Si bien para Proust habría una similitud o incluso una igualdad entre la magdalena de ambos presentes, Deleuze afirma que, dado que la reminiscencia es involuntaria, el 
sabor tiene un "poder" que lo hace conllevar el elemento paradójico, y con ello independizarse del presente pasado y del presente presente. La magdalena y Combray se presentan al protagonista como un recuerdo puro. Este pasado puro nunca fue presente, pero aun así alcanza intempestivamente al narradory lo hace expresarlo, que no es otra cosa que escribir En busca del tiempo perdido.

El punto de todo esto es que, para Deleuze, así como la obra no es una representación de alguno de los presentes mencionados, tampoco es una representación del pasado puro, es decir, es independiente de él. El sabor de la magdalena, en cuanto acontecimiento, se elevó a nivel de incorporal (pasado puro que no toca ningún presente) y produjo una obra en el futuro que, sin embargo, es independiente de él. El sentido, como he venido diciendo, funda pero no se asemeja a lo que funda.

\section{§ 5. Conclusiones}

Uno de los problemas con los que uno debe enfrentarse al leer a Deleuze es la brecha entre su clara influencia fenomenológica y su gran desarraigo del lenguaje fenomenológico. Hablar aquí de lenguaje implica, por supuesto, hablar de visión o, en sus términos, de imagen del pensamiento, lo cual eleva las diferencias entre ambos autores desde lo terminológico a lo propiamente filosófico y de interés primordial. Es común hablar del exceso de términos técnicos e inoportunos en Husserl, así como de la poca previsibilidad -y con ello quizá hermetismo- del razonamiento de Deleuze. Ninguno de ambos tópicos me parece casual. Si bien esto no está tan claro desde su propio paradigma, tengo el convencimiento de que un asunto de términos en Husserl es un asunto de pensamiento, un problema de recorrido fenomenológico que afecta, curiosamente, los propios cimientos de la fenomenología. Tengo también el convencimiento de que las críticas de Deleuze son audaces, y por otro lado podrían no ser consideradas totalmente justas, en vista de que no parece tener las mismas expectativas que Husserl con respecto de la filosofía. Parece ser, pues, que mientras que uno intenta fundar las condiciones para un conocimiento riguroso sin hacer metafísica, el otro lleva consigo la impronta creativa nietzscheana que obliga a intuir cuáles serán los siguientes intersticios que pueda recorrer el pensamiento humano, más allá de si nos brinda un conocimiento organizado o no. 
Este trabajo no ha sido más sobre Deleuze que sobre Husserl, puesto que me he limitado a explicitar los puntos compartidos, si bien con un apego mayor a los intereses del primero, con el fin de trazar una lectura transversal a un elemento que puedo decir está en ambos, y en general, en la filosofía contemporánea: la noción de estructura. No es casualidad que la noción de sentido haya surgido con el abandono del pensamiento substantivo y el advenimiento de planteamientos que establecen relaciones como premisas fundamentales. Es el caso de Husserl, para quien la relación intencional fundamenta la multiplicidad de la experiencia y con ello la condición de posibilidad de un conocimiento del mundo. También es el caso de Deleuze, para quien el sentido es un decàlage, un desequilibrio perpetuo estructural que flota entre los cuerpos y los incorporales (o las cosas y el lenguaje). Si algo puedo rescatar de este autor es el intento de llevar la estructura al terreno de la diferencia y el movimiento, no solo fuera del terreno platónico, sino lejos de él. Aquí lo propiamente platónico no es el mundo inteligible sino, como, dice Deleuze, la noción de que si no hay orden, hay caos, y por tanto la idea de que la tarea fundamental de una estructura es establecer un orden centrado, un lineamiento con sentido.

Después de todo, la impronta fenomenológica (que Deleuze no podrá negar) incluye ya desde Husserl una cláusula de inacabamiento, de posibilidad de síntesis comunitaria. ¿Y si ese camino conllevara un descentramiento? ¿Una crítica a la estructura de la intencionalidad? Me parece que, como sucede con los grandes filósofos, las intuiciones base de su filosofía son materia de su propia crítica, y al mismo tiempo no hay crítica válida a su pensamiento que prescinda de sus intuiciones. 